\title{
Observing Directional Properties of Ocean Swell with an Acoustic Doppler Current Profiler (ADCP)
}

\author{
T. H. C. HERBERS \\ Department of Oceanography, Naval Postgraduate School, Monterey, California \\ S. J. LENTZ \\ Woods Hole Oceanographic Institution, Woods Hole, Massachusetts
}

(Manuscript received 3 December 2008, in final form 2 July 2009)

\begin{abstract}
Acoustic Doppler current profilers (ADCPs) are widely used for routine measurements of ocean currents and waves in coastal environments. These instruments have the basic capability to measure surface wave frequency-directional spectra, but the quality of the estimates is not well understood because of the relatively high noise levels in the velocity measurements. In this study, wave data are evaluated from two $600-\mathrm{kHz}$ ADCP instruments deployed at 20- and 45-m depths on the Southern California continental shelf. A simple parametric estimation technique is presented that provides robust estimates of the gross directional wave properties, even when the data quality is marginal, as was often the case in this benign wave environment. Good agreement of mean direction and (to a lesser degree) directional spreading estimates with measurements from a nearby surface-following buoy confirms that reliable wave information can generally be extracted from ADCP measurements on the continental shelf, supporting the instrument's suitability for routine wave-monitoring applications.
\end{abstract}

\section{Introduction}

The acoustic Doppler current profiler (ADCP) is the most widely used instrument for observing ocean currents. Many studies have demonstrated the unique capabilities of this technique (based on sampling the range-gated Doppler return of acoustic beams); it can map the detailed space-time evolution of internal waves (e.g., Pinkel 1979, 1983) and small-scale nearshore circulation features (Smith 1993; Smith and Largier 1995), and it can resolve the directional properties of ocean surface waves (Pinkel and Smith 1987; Krogstad et al. 1988). Whereas early ADCP systems, because of their cost, size, and power requirements, were used primarily in basic research investigations, the recent development of commercially available compact, self-contained instruments with low power requirements has led to widespread use of the ADCP in routine coastal applications, such as the moni-

Corresponding author address: Dr. Thomas H. C. Herbers, Department of Oceanography, Naval Postgraduate School, Monterey, CA 93943.

E-mail: thherber@nps.edu toring of tidal and wind-driven currents and sea and swell waves.

The most common configuration of ADCP instruments is the so-called Janus configuration consisting of four acoustic transducers that look upward (or downward) at a small inclination in azimuthally orthogonal directions. The along-beam velocity profiles are obtained by transmitting acoustic pulses and measuring the Doppler shift in the range-gated backscatter return caused by small natural particles in the water column that are advected by the flow. The first-generation, so-called narrowband ADCPs relied on pulse-to-pulse incoherent processing, resulting in noise levels that were too high to resolve high-frequency wind waves and swell, and thus these instruments were used primarily to observe mean current profiles based on long averaging intervals. On the other hand, pulse-to-pulse coherent systems (Lhermitte and Serafin 1984) with very low noise levels can provide accurate measurements of the wave orbital flow field (e.g., Herbers et al. 1991, 1992) but suffer from a rangevelocity ambiguity that is undesirable for long-range current profiling applications. The second-generation of so-called broadband ADCPs combines features of 
incoherent and coherent sonars to reduce noise levels while maintaining the full-range profiling capability (Brumley et al. 1991). This instrument thus allows for simultaneous measurements of mean current profiles and surface wave spectra, and it is now widely used for coastal wave-monitoring and current-monitoring applications (e.g., Terray et al. 1999; Work 2008).

The ADCP velocity time series, collected at a large number of positions spanning the horizontal aperture of the four beams, contain detailed two-dimensional phase information of the surface wave field. These multicomponent observations can be analyzed using cross-spectral array processing techniques to infer the frequencydirectional surface wave spectrum (Terray et al. 1999). Although the large number of array elements (typically 24 when burst samples are collected at six vertical levels) can in principle resolve the detailed structure of the directional wave energy distribution, this capability is in practice compromised by the relatively high noise level of the velocity measurements and the limited horizontal aperture of the array (less than the water depth for the typical $20^{\circ}-30^{\circ}$ vertical inclination of the ADCP beams). High-resolution estimation techniques that yield an exact or approximate fit to array cross-spectra are known to be sensitive to measurement errors, often resulting in spurious structure in the directional spectrum estimates (e.g., Long and Hasselmann 1979; Pawka 1983; Herbers and Guza 1990). In addition to the high noise level of the velocity measurements, the ADCP sampling (in upwardlooking configuration) is sparse near the surface and may not adequately resolve the high-frequency wave motion that is confined to the upper part of the water column. For these relatively short-wavelength waves, the near-surface velocities measured by the different beams are incoherent and only a limited set of array lags within the same beam are available to resolve the wave directional properties. On the other hand, relatively long-wavelength (compared with the water depth) swells are oversampled by the ADCP, and the resolving power is limited by the horizontal array aperture that spans only a fraction of a wavelength. How these limitations affect the quality of ADCP directional wave measurements is not well understood.

In this study, the accuracy of ADCP directional wave measurements is evaluated using data from two RDI 600-kHz ADCPs deployed on the inner continental shelf off the southern California coast. The experiment and data collection are described in section 2. To obtain robust estimates of the gross directional wave properties in this benign wave environment where the signal-to-noise ratio is often marginal, a simple parametric technique is presented in section 3. The directional distribution at each frequency is obtained by fitting a unimodal or bimodal cosine-power shape to the full cross-spectral matrix. This technique was chosen over more sophisticated inverse methods, because for many applications knowing the mean propagation direction and spreading of the dominant wave systems is often sufficient and inverse model estimates with more degrees of freedom obtained from these inherently noisy datasets often contain spurious features that require cautious interpretation. The method presented here allows for a direct evaluation of the best-fit solution by sweeping through the parameter space, thus avoiding the iterative approach that is needed to solve the nonlinear inverse problem (e.g., Long and Hasselmann 1979; Pawka 1983; Herbers and Guza 1990). The accuracy of the ADCP directional spectrum estimates is evaluated in section 4 through comparison with independent estimates from a surface-following buoy. In general, good agreement with the buoy observations throughout the experiment with low to moderately energetic swell conditions confirms that the ADCP is a robust directional wave sensor that can provide reliable routine wave information. Although the mean wave direction as a function of frequency is accurately resolved in the ADCP measurements, estimates of directional spreading are biased high in low wave conditions. Monte Carlo simulations presented in section 5 demonstrate that this bias can be largely explained by the relatively high instrument noise levels of the ADCP. The results are summarized in section 6 .

\section{Field data}

Two ADCPs were deployed on the continental shelf near La Jolla, California, during the fall of 2003 as part of the Nearshore Canyon Experiment (NCEX; Magne et al. 2007; Thomson et al. 2007). Although the focus of NCEX was on the effects of the La Jolla and Scripps submarine canyons on the wave field, the ADCPs were located on an approximately alongshore uniform section of the shelf, well to the north of the canyons (Fig. 1). The $600-\mathrm{kHz}$ Teledyne Sentinel Workhorse ADCPs were deployed in 20- ("shallow ADCP") and 45-m ("deep ADCP") depths (Fig. 1) on bottom tripods, looking vertically upward with the transducer head about $0.6 \mathrm{~m}$ above the seafloor. The maximum range of the instruments is about $50 \mathrm{~m}$, thus allowing velocity profile measurements that span the water column at both sites.

The ADCP contains four beams that look upward in a Janus configuration at a $20^{\circ}$ angle relative to the vertical. At both the 20- and 45-m depth sites, velocities were sampled over almost the entire water column with 1 - $\mathrm{m}$ vertical bin resolution and a 0.88 -m blanking distance. The ADCP instruments were operated in a standard dualprofiling and wave-burst sampling mode. Mean velocity 


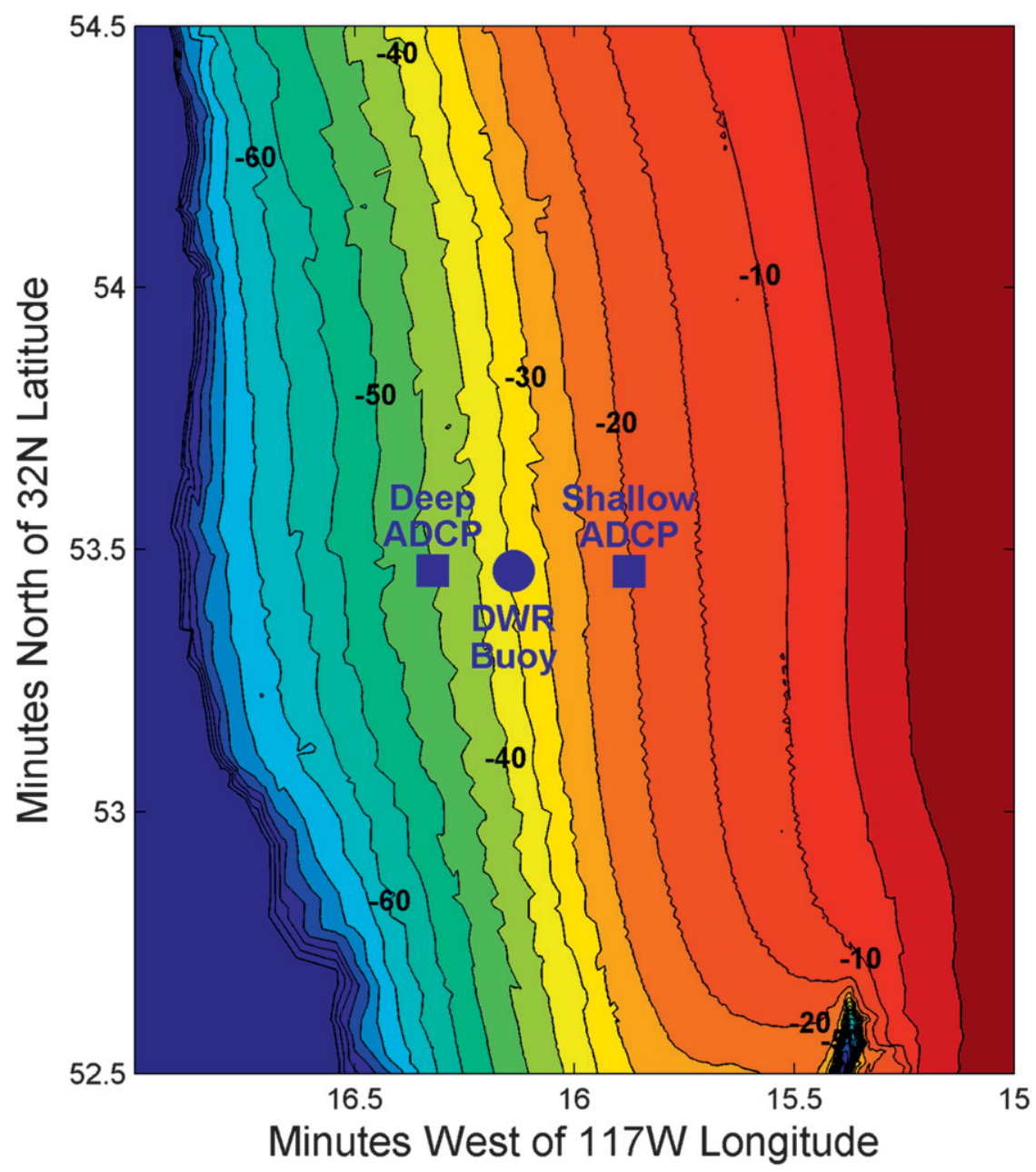

FIG. 1. Locations of three instruments deployed near La Jolla, California, during the fall of 2003 as part of NCEX: the deep ADCP in 45-m depth, the shallow ADCP in 20-m depth, and in between the DWR buoy in 35-m depth. Depth contours are shown at 5-m intervals. The dark blue area offshore indicates depths greater than $100 \mathrm{~m}$, and the dark red area inshore indicates dry land. The bathymetry feature in the lower right corner is the head of Scripps Canyon, which does not significantly affect the wave field at the three instruments located well to the north.

profiles were collected continuously at 15 -min intervals using an average of 200 pings. Wave bursts of 8192 pings at 0.5 -s intervals (i.e., a 68-min-long record) were collected every $3 \mathrm{~h}$. The wave bursts sampled six selected depth cells for all four beams to yield a spatial array of 24 measurements. The selected depth cells were distributed over the water column to examine the variation in data quality at different ranges and achieve a dense array geometry with the maximum aperture. At the 20-m depth site, burst samples were collected at bins 2, 5, $8,11,13$, and 14 (counted from the nearest bin to the transducer), corresponding to heights above the seafloor of 3.4, 6.2, 9.1,11.9, 13.8, and $14.7 \mathrm{~m}$, respectively. At the 45-m depth site, the selected bins $(2,10,18,26,34$, and
42) were at heights above the seafloor of $3.4,10.9,18.5$, $26.0,33.5$, and $41.0 \mathrm{~m}$. The wave-burst data also contain a pressure record collected with the same sampling scheme. The ADCP pressure gauge is located near the acoustic transducer head, about $0.6 \mathrm{~m}$ above the seafloor.

A surface-following buoy was located between the two ADCP sites in about 35-m depth (Fig. 1). The buoy is a 0.9 -m-diameter Datawell Directional Waverider (DWR) buoy equipped with a three-component Hippy accelerometer package, compass, and tilt sensors. It provides time series of horizontal $(x, y)$ and vertical $(z)$ displacements of the buoy from which the surface wave spectrum and standard directional moments can be extracted (e.g., Kuik et al. 1988). The accuracy of this 
buoy has been established in earlier studies (e.g., O'Reilly et al. 1996), and it is believed to provide reliable estimates of surface elevation spectra and of mean propagation directions and directional spreading (as a function of frequency). Although not a perfect "ground truth," the buoy observations provide an independent verification that may be useful to identify possible discrepancies in the ADCP estimates. The buoy collected continuous $x-y-z$ displacement data with a sample frequency of $1.28 \mathrm{~Hz}$.

This study uses field data from a 7-week period, 27 October-12 December 2003, when both ADCP instruments were deployed. Concurrent Waverider buoy data were collected from 27 October to 1 December. ADCP-buoy comparisons were conducted for this shorter 5-week period.

Wave conditions at the study site are dominated by long-period (nominally 12-18 s) Pacific swell. The data collection took place in the fall, which is typically a transition period between the summer season that is dominated by Southern Hemisphere swells and the more energetic winter season with swells from North Pacific storms (e.g., Munk et al. 1963; Pawka 1983). Local winds, measured at the nearby Scripps Pier (courtesy of the Coastal Data Information Program), were weak throughout the experiment (sustained speeds less than $5 \mathrm{~m} \mathrm{~s}^{-1}$ ), with the exception of a moderate $\left(7-8 \mathrm{~m} \mathrm{~s}^{-1}\right)$ wind event that occurred toward the end of the experiment (11-12 December), when the Waverider buoy was no longer in operation. The tidal range (measured with the ADCP pressure gauge) varied from 1.0 to $2.7 \mathrm{~m}$, causing minor depth variations that were taken into account in the analysis. ADCP observations of the mean alongshore flows show a predominant semidiurnal internal tide contribution with amplitudes varying from 5 to $15 \mathrm{~cm} \mathrm{~s}^{-1}$, whereas the cross-shore mean flows indicate a broad internal wave spectrum with similar magnitudes. These relatively weak background currents do not significantly affect the long-period swell surface wave field at this site and are neglected here.

The ADCP velocity data were screened for occasional dropouts caused by, for example, obstruction of the acoustic beams by passing fish or a lack of scatterers in the water column. In most cases, dropouts occurred for isolated data samples or a few consecutive data samples that could be readily corrected through interpolation of the adjacent good velocity samples. Wave bursts that contained more than $2 \%$ bad data or a dropout lasting more than $5 \mathrm{~s}$ were discarded. This screening resulted in 369 accepted and 1 rejected wave bursts at the shallow (20-m depth) ADCP. Data from the deep (45-m depth) ADCP contained more dropouts, resulting in 306 accepted and 63 rejected wave bursts.

\section{Analysis technique}

The ADCP wave-burst velocity measurements collected along four beams at six range bins constitute a dense 24-element array with detailed directional wave information. However, the resolving power of this array is limited by the horizontal aperture (about 13 and $30 \mathrm{~m}$ for the shallow and deep ADCP, respectively) and the relatively high noise level of the velocity measurements. The purpose of this study is to assess whether the ADCP can provide reliable estimates of the gross directional wave properties, such as the mean direction and directional spreading of the dominant wave systems, which are of primary interest in routine wave-monitoring applications. A simple and robust technique for the array analysis is presented here, based on a frequently used parametric model of the directional distribution of wave energy in the form of a unimodal or bimodal cosine-power shape. Fitting such a model with a small number of degrees of freedom to the array cross-spectra has the advantage of avoiding estimates with a potentially spurious structure that would require cautious interpretation. Furthermore, in cases of very low signal-to-noise ratios, where more sophisticated inverse methods may be unstable or yield unphysical solutions, the simpler parametric approach is expected to be more robust and hopefully identify at least the dominant wave direction in the noisy dataset.

\section{a. General definitions}

The surface elevation function $\eta$ of a random, homogeneous wave field can be expressed as a superposition of plane waves with different frequencies $\omega$ and propagation directions $\theta$ :

$$
\eta(\mathbf{x}, t)=\sum_{\omega} \sum_{\theta} A_{\omega, \theta} \exp [i(\mathbf{k} \cdot \mathbf{x}-\omega t)] .
$$

The wavenumber vector in (1) is defined as $\mathbf{k}=s_{ \pm}(k \cos \theta$, $k \sin \theta)$, with $k \equiv|\mathbf{k}|$ obeying the linear gravity wave dispersion relation $\omega^{2}=g k \tanh (k h)$, where $g$ is gravity and $h$ is the water depth, and the sign index $s_{ \pm}$is +1 for positive $\omega$ and -1 for negative $\omega$. The complex amplitudes obey the symmetry relation $A_{-\omega, \theta}=\left(A_{\omega, \theta}\right)^{*}$, where the asterisk denotes the complex conjugate.

The sea surface amplitudes $A_{\omega, \theta}$ are assumed to be statistically independent. In the limit of small separation of the frequencies $\Delta \omega$ and directions $\Delta \theta$, their statistics can be described by a continuous spectrum,

$$
\left\langle\left|A_{\omega, \theta}\right|^{2}\right\rangle \equiv E(\omega, \theta) \Delta \omega \Delta \theta,
$$

where the angle brackets denote the expected value. It follows from (1) and (2) that the integral of the frequency-directional spectrum $E(\omega, \theta)$ over all frequencies and directions equals the surface elevation variance 


$$
\left\langle\eta^{2}\right\rangle=\int_{-\infty}^{\infty} d \omega \int_{0}^{2 \pi} d \theta E(\omega, \theta)
$$

\section{b. The inverse problem}

The ADCP velocity measurements are related to the sea surface elevation (1) through linear transfer functions:

$$
V_{n}^{m}(t)=\sum_{\omega} \sum_{\theta} G_{n}^{m}(\omega, \theta) A_{\omega, \theta} \exp (-i \omega t)
$$

where the subscript $n$ indicates the beam number (1-4) and the superscript $m$ is the depth cell index (1-6). According to linear theory, the transfer function $G_{n}^{m}$ is given by

$$
G_{n}^{m}(\omega, \theta)=\frac{g k \exp \left(i \mathbf{k} \cdot \mathbf{x}_{n}^{m}\right)}{\omega \cosh (k h)}\left[\sin \alpha \cosh \left(k d_{m}\right) \cos \left(\theta-\theta_{n}\right)-i \cos \alpha \sinh \left(k d_{m}\right)\right]
$$

where $\mathbf{x}_{n}^{m}$ is the horizontal position vector of the cell, $d_{m}$ is the height above the seafloor, $\theta_{n}$ is the orientation of beam $n$ in the horizontal plane relative to the $x$ axis, and $\alpha$ is the angle of the beams relative to the vertical.

The covariance of any pair of velocity measurements can be written as [using (2) and (4)]

$$
\begin{aligned}
\left\langle V_{n}^{m}(t) V_{r}^{s}(t)\right\rangle & \equiv \int_{-\infty}^{\infty} d \omega C_{V_{n}^{m} V_{r}^{s}}(\omega) \\
& =\int_{-\infty}^{\infty} d \omega \int_{0}^{2 \pi} d \theta G_{n}^{m}(\omega, \theta)\left[G_{r}^{s}(\omega, \theta)\right]^{*} E(\omega, \theta),
\end{aligned}
$$

yielding an expression for the cross-spectrum $C_{V_{n}^{m} V_{r}^{s}}(\omega)$ :

$$
C_{V_{n}^{m} V_{r}^{s}}(\omega)=\int_{0}^{2 \pi} d \theta G_{n}^{m}(\omega, \theta)\left[G_{r}^{s}(\omega, \theta)\right]^{*} E(\omega, \theta) .
$$

Equation (6) defines the relationship between the unknown frequency-directional spectrum $E(\omega, \theta)$ and the observed cross-spectra $C_{V_{n}^{m} V_{r}^{s}}(\omega)$.

\section{c. The frequency spectrum}

It is convenient to decompose the two-dimensional wave spectrum in a frequency spectrum $E(\omega)$ and a directional distribution at each frequency $S(\theta ; \omega)$,

$$
E(\omega ; \theta) \equiv E(\omega) S(\theta ; \omega)
$$

with

$$
\int_{0}^{2 \pi} d \theta S(\theta)=1
$$

Because of the orthogonal beam configuration, the sum of the autospectra is independent of direction and yields a direct estimate of the wave frequency spectrum:

$$
\hat{E}(\omega)=\frac{\omega^{2} \cosh ^{2}(k h) \sum_{n} \sum_{m} C_{V_{n}^{m} V_{n}^{m}}(\omega)}{g^{2} k^{2} \sum_{m}\left[2 \sin ^{2} \alpha \cosh ^{2}\left(k d_{m}\right)+4 \cos ^{2} \alpha \sinh ^{2}\left(k d_{m}\right)\right]} .
$$

Other combinations of the autospectra can be used to estimate $E(\omega)$. The use of equal weights in (9) has the advantage that it tends to reject velocity measurements with low signal-to-noise ratios. For short-wavelength, high-frequency waves, the estimate (9) is dominated by the larger signals in the upper cells and thus is not seriously degraded by the noisy lower cells. On the other hand, for long-wavelength waves with relatively weak vertical motions and horizontal flows that are uniform over the water column, all depth cells contribute equally to (9), yielding a robust estimate of $E$ that uses all measurements. Hoitink et al. (2007) present a more sophisticated method for estimating $E(\omega)$ that filters out incoherent noise in the ADCP velocity measurements. Alternatively, $E(\omega)$ can also be estimated from the pressure record of the ADCP, which has relatively low noise levels but, because it is collected near the seafloor, is more attenuated at high frequencies than the velocity measurements collected higher up in the water column. Comparing estimates of $E(\omega)$ based on (9) with the independent pressure-based estimates provides a useful check of the overall quality of the velocity measurements that may identify excessive instrument noise levels or contamination with nongravity wave flow contributions (e.g., Lippmann et al. 1999; Hoitink et al. 2007).

\section{d. The directional distribution}

Normalizing (6) by the frequency spectrum estimate $\hat{E}$ [using (7)] yields a relation between the observed ADCP velocity cross-spectra and the unknown directional distribution of wave energy $S(\theta)$. This set of equations can be written compactly as 


$$
\int_{0}^{2 \pi} d \theta \mathbf{g}(\theta) S(\theta)=\mathbf{d},
$$

where the elements of vector $\mathbf{d}$ are the normalized crossspectra $C_{V_{n}^{m} V_{r}^{s}} / \hat{E}$, and the elements of vector $\mathbf{g}$ contain the products ${ }^{r} G_{n}^{m}\left(G_{r}^{s}\right)^{*}$ of the corresponding transfer functions. Including all auto- and cross-spectra of the 24 velocity time series provided by the ADCP (six bins are sampled for each of the four beams), the data vector d has 300 elements. Although there is redundancy (e.g., the autospectra of opposing beams), this large dataset can in principle yield high-resolution estimates of $S(\theta)$. However, in practice, this capability is compromised by the small horizontal aperture of the array and high noise levels of the ADCP velocity measurements. Highresolution estimation techniques that attempt to fit an exact or near-exact solution to (10) are known to be extremely sensitive to errors in the observations (especially for wavelengths that are long compared with the array footprint), typically resulting in estimates of $S(\theta)$ with wildly spurious structures (see Herbers and Guza 1990 for further discussion and examples).

For most routine applications, knowing the detailed structure of $S(\theta)$ is not critical and estimates of the mean direction and spread of the dominant wave systems are often sufficient. Robust estimates of these gross directional properties can be extracted from the large but noisy dataset by prescribing a parametric model $\hat{S}(\theta)$ with a small number of free parameters and finding the set of parameter values that optimize the fit of $\hat{S}(\theta)$ to the observations (10). A frequently used parametric model is a simple cosine-power function of the form

$$
\hat{S}(\theta)=c_{N} \cos ^{2 s}\left(\frac{\theta-\bar{\theta}}{2}\right),
$$

where $\bar{\theta}$ is the mean wave direction, the parameter $s$ controls the width of the distribution, and $c_{N}$ is a normalization constant (e.g., Longuet-Higgins et al. 1963; Mitsuyasu et al. 1975; Hasselmann et al. 1980). The directional spread $\sigma$, defined here as the half-width of the directional distribution at half-maximum power, is related to $s$ by

$$
s=\frac{\log (1 / 2)}{\{2 \log [\cos (\sigma / 2)]\}} .
$$

The unimodal form (11) is readily extended to a doublepeaked function that allows for the representation of a bimodal wave field, such as a wind sea in the presence of swell (e.g., Hasselmann et al. 1980),

$$
\hat{S}(\theta)=c_{N 1} \cos ^{2 s_{1}}\left(\frac{\theta-\bar{\theta}_{1}}{2}\right)+c_{N 2} \cos ^{2 s_{2}}\left(\frac{\theta-\bar{\theta}_{2}}{2}\right) .
$$

The free parameters of $\hat{S}$ [either the unimodal form (11) or the bimodal form (13)] can be estimated by fitting the distribution to the observed cross-spectra (10). To quantify the goodness of fit, a misfit $\varepsilon$ is defined as

$$
\boldsymbol{\varepsilon} \equiv \mathbf{d}-\int_{0}^{2 \pi} d \theta \mathbf{g}(\theta) \hat{S}(\theta) .
$$

An optimal model $\hat{S}$ that best fits the observations is obtained by selecting the set of parameters $\left(c_{N i}, \bar{\theta}_{i}, s_{i}\right)$ that minimizes the $l^{2}$ norm $|\boldsymbol{\varepsilon}|=\sqrt{\varepsilon \cdot \boldsymbol{\varepsilon}}$. Because the number of free parameters is small [two for the unimodal distribution (11) and five for the bimodal distribution (13); one of the normalization constants $c_{N i}$ follows from the unit integral constraint (8)], a global minimum of $|\varepsilon|$ can be readily determined by evaluating $\varepsilon$ for all possible combinations of the free parameters. Here, solutions were evaluated for 60 values of $\bar{\theta}_{i}$ spanning the full circle at $6^{\circ}$ increments, 10 values of $s_{i}$ corresponding to spread values [Eq. (12)] increasing at an equal increment factor of 1.4 from $5^{\circ}$ to $90^{\circ}$, and (for the bimodal distribution) 10 variance ratios (between the minor and major peak) varying from 0.05 to 0.5 at equal intervals of 0.05 .

\section{Results}

Estimates of the frequency-directional spectrum $E(\omega, \theta)$ were extracted from each 68-min ADCP wave burst using the parametric technique described in the previous section. The array cross-spectra were computed based on 128 -s segments with $50 \%$ overlap. After removing the mean and applying a Hamming window, the segments were Fourier transformed and (ensemble averaged) cross-spectra with a bandwidth $\Delta \omega / 2 \pi$ of $0.0078 \mathrm{~Hz}$ were computed for the frequency range $\omega / 2 \pi=0.04-0.19 \mathrm{~Hz}$ that contains the dominant swell energy. The lowfrequency limit was chosen to exclude infragravity motions that contain second-order nonlinear bound wave contributions (e.g., Herbers et al. 1994) and possibly other (nongravity wave) fluid motions (e.g., Lippmann et al. 1999) that do not obey the linear theory transfer function, Eq. (5). Frequencies higher than $0.19 \mathrm{~Hz}$ were not considered, because the relatively weak energy levels and strong vertical decay of these short-wavelength waves resulted in marginal data quality (i.e., low signal-to-noise ratios) for the deep ADCP (see Fig. 6 discussed below).

Two independent estimates of the frequency spectrum were computed:1) a "velocity based" estimate that applies a linear transfer function to the sum of the velocity spectra [Eq. (9)] and 2) a "pressure based" estimate that applies a linear theory transfer function to the observed near-bottom pressure spectrum. In the 
intermediate depths and benign, swell-dominated environment of these measurements, linear theory accurately predicts the wave-induced pressure and orbital velocity field (e.g., Herbers et al. 1992), and thus these independent estimates provide a consistency check on the ADCP velocity measurements that does not depend on the unknown directional properties of the wave field. For convenience, the results presented here use the singlesided frequency spectrum definition $E(f) \equiv 4 \pi E(\omega)$, where $f=\omega / 2 \pi$ is the frequency in hertz.

At each frequency, an estimate of the directional distribution of wave energy was obtained by fitting the parametric cosine-power functions to the array crossspectra. Optimal distributions with both a unimodal shape [Eq. (11)] and a bimodal shape [Eq. (13)] were evaluated by selecting the model parameters that minimize the misfit norm $|\boldsymbol{\varepsilon}|[$ Eq. (14)]. The initial trials indicated that the resulting minimal misfit values for a bimodal distribution were not significantly lower than those for the unimodal distribution. This is not surprising, considering the close proximity of a dissipative shoreline (less than $2 \mathrm{~km}$ ) and the sheltering effects of offshore islands (see Pawka 1983) that restrict wave arrivals to a narrow range of southwesterly to westerly directions (that are further reduced by refraction over the continental shelf). Although the simultaneous arrival of multiple swell systems is not uncommon at this site, the directional spreading angle is apparently too small to resolve the two peaks at the same frequency in the ADCP velocity measurements. In the following analysis, results for the unimodal distribution model are presented that are defined by only two parameters: the mean wave direction $\bar{\theta}$ and the directional spread $\sigma$ (the half-width of the directional distribution at half-maximum power). Estimates of the surface height frequency spectrum $E(f)$ and of the mean direction $\bar{\theta}(f)$ and directional spread $\sigma(f)$ (both functions of frequency) in the frequency range $0.04-0.19 \mathrm{~Hz}$ were obtained for all wave bursts collected by the two ADCP instruments.

The analysis of the Waverider buoy data was performed using a conventional method to estimate the mean wave direction $\bar{\theta}$ and the directional spread $\sigma$ from the cospectra of the horizontal and vertical buoy displacement time series (e.g., Kuik et al. 1988). Although these parameters, based on the lowest Fourier moments of $S(\theta)$, differ from the definitions of $\bar{\theta}$ and $\sigma$ used here in the ADCP analysis, the bias is negligible (see appendix in Herbers et al. 1999) for the directionally narrow $\left(\sigma<30^{\circ}\right)$ wave fields observed in this study. Because of the different sampling scheme, the buoy analysis was performed on 70-min-long records (that fully contain the 68 -min interval of the ADCP wave burst), broken into $50 \%$ overlapping 200 -s segments. The ensemble- averaged cross-spectra (again using a Hamming window) have a bandwidth of $0.005 \mathrm{~Hz}$. Estimates of the surface height frequency spectrum $E(f)$ and of the mean direction $\bar{\theta}(f)$ and directional spread $\sigma(f)$ were computed in the same frequency range $(0.04-0.19 \mathrm{~Hz})$ as the ADCPbased estimates.

To compare the ADCP- and buoy-based estimates of wave spectra and directional parameters, the shoaling and refraction effects induced by spatial depth variations need to be taken into account. Over the relatively short propagation distance (about $0.7 \mathrm{~km}$ ) from the deep to the shallow ADCP, dissipation caused by bottom friction is expected to be negligible (e.g., Ardhuin et al. 2003, and references therein); for the approximately alongshore uniform shelf, the effects of shoaling and refraction are well described by Snell's law and the conservation of the cross-shore energy flux. For small wave incidence angles, the refraction effect on the wave energy can be neglected and the conservation of energy flux relation reduces to $E(f) c_{g}(f)=$ constant, where $c_{g}$ is the linear wave group speed. For small wave incidence angles, the shape of the directional distribution is preserved and the refraction transformation of $\bar{\theta}(f)$ and $\sigma(f)$ are governed by the simple relations $\left[\bar{\theta}(f)-\theta_{n}\right] / c(f)=$ constant and $\sigma(f) / c(f)=$ constant, where $c$ is the linear wave phase speed and $\theta_{n}$ is the angle of normal incidence. Here, the ADCP-based estimates of $E(f), \bar{\theta}(f)$, and $\sigma(f)$ were transformed to the Waverider buoy location using an approximate $\theta_{n}=260^{\circ}$ shore-normal direction. The observed mean wave directions deviate by less than $30^{\circ}$ from this normal incidence angle (see Fig. 2c discussed below), and thus the small angle approximation is reasonable for the present dataset. The shoaling and refraction corrections are generally small; the maximum predicted shoaling change in spectral energy levels is about $20 \%$ (i.e., a $10 \%$ change in wave height), and the maximum refraction change in wave incidence angle is about $25 \%$.

Estimates of bulk wave parameters obtained from the ADCP velocity measurements are compared in Fig. 2 to estimates extracted from the Waverider buoy data. The significant wave height $H_{s} \equiv 4 \sqrt{E}$, where $E$ is the surface elevation variance, was estimated by integrating the surface height spectra $E(f)$ (shoaled to the Waverider buoy location) over the swell-sea frequency range 0.04 $0.19 \mathrm{~Hz}$. Estimates of $H_{s}$ based on the ADCP velocity measurements are generally in good agreement with the Waverider estimates throughout the experiment. However, estimates from the deep ADCP show a small but persistent positive bias (about $10 \%-20 \%$ ) suggesting an overestimation of wave energy levels that is (as discussed further below) probably caused by elevated noise levels in the velocity spectra. In comparison, the shallow 

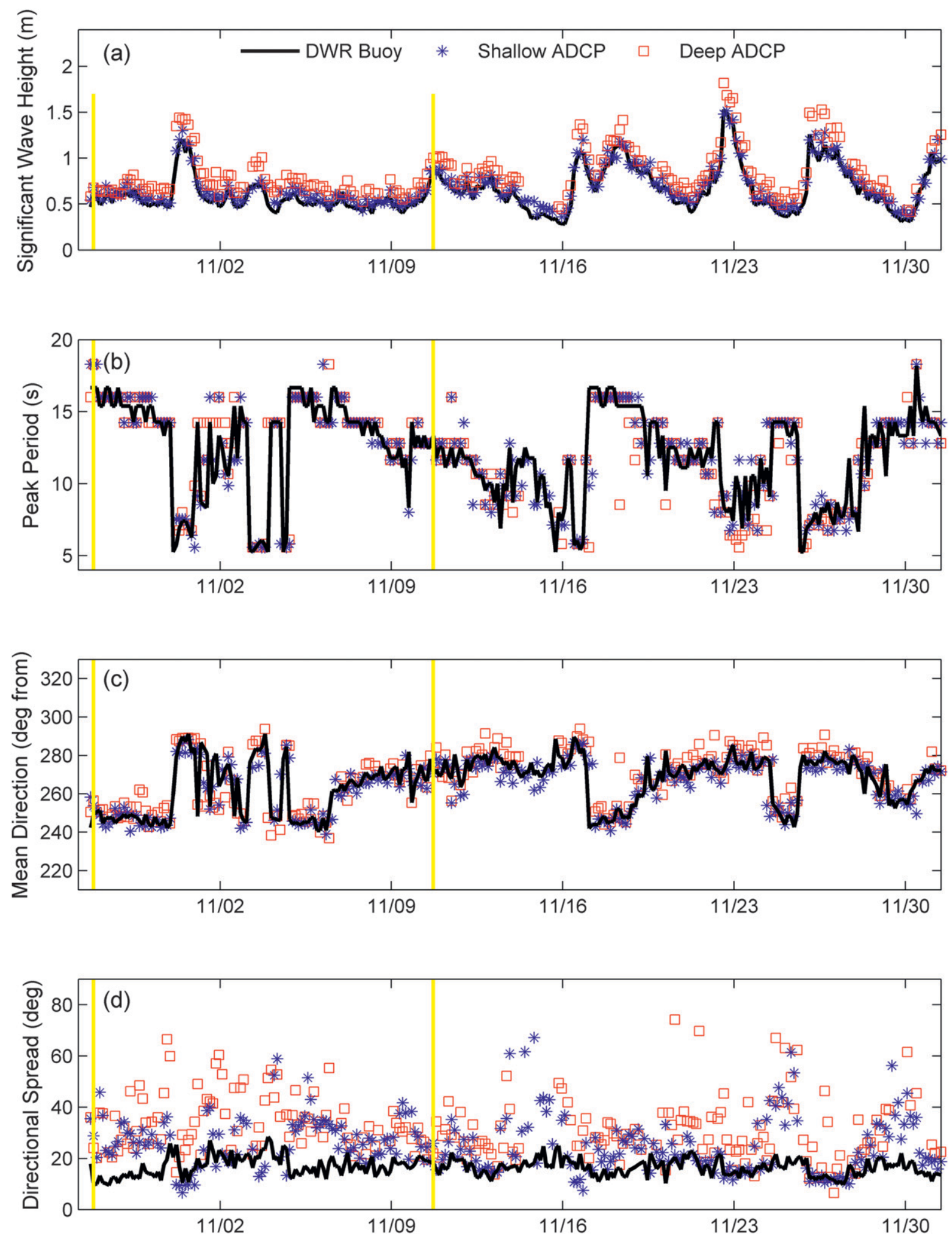

FIG. 2. Comparison of ADCP and Waverider buoy estimates of bulk wave statistics: (top)-(bottom) significant wave height, spectral peak period, mean wave direction, and directional spread (both at the peak frequency). Solid black curves indicate estimates from the Waverider buoy. Symbols denote estimates from the two ADCPs, transformed to the buoy depth using a frequency-dependent linear theory correction for shoaling and refraction effects. The vertical yellow lines indicate the times of the two case studies detailed in Figs. 4 and 5. 
ADCP estimates are in excellent agreement with the Waverider estimates, with the exception of a few periods with very low $(<0.5 \mathrm{~m})$ wave heights (e.g., 14-16 November) where the ADCP-based estimates are biased high by about $10 \%-20 \%$.

ADCP and Waverider estimates of the wave peak period $T_{p}$ [the period corresponding to the peak frequency $f_{p}$ of $E(f)$ ] are in good agreement (Fig. 2b). Estimates of the mean direction $\bar{\theta}_{p}$ and directional spread $\sigma_{p}$ at the peak frequency (an energy-weighted average of a $0.025-\mathrm{Hz}$ bandwidth centered at $f_{p}$ ) are compared in Figs. 2c,d, respectively. At both ADCP sites, estimates of $\bar{\theta}_{p}$ agree well with the Waverider estimates (Fig. 2c). Differences are generally within the $\pm 5^{\circ}$ uncertainty of the instrument compasses. These results indicate that noise in the velocity measurements does not significantly bias the ADCP estimates of the dominant wave direction. On the other hand, comparisons of $\sigma_{p}$ show significant differences between the ADCP and Waverider estimates (Fig. 2d). Whereas the Waverider directional spreading estimates are in the range of $10^{\circ}-25^{\circ}$, the ADCP estimates are often much higher (up to $70^{\circ}$ ). The Waverider estimates are consistent with the wave climate at this site that is dominated by remotely generated swell with narrower spreading than is common in locally generated seas (Pawka 1983), and the accuracy of Datawell Waverider estimates of directional spreading has been verified through comparisons with fixed platform array measurements of Pacific swell (O'Reilly et al. 1996). Hence, the present results indicate that the ADCP directional spreading estimates are biased high. It should be noted that the agreement is generally better for more energetic wave conditions, for example around 22-23 and 26-27 November, especially for the shallow ADCP, suggesting that a lower signal-to-noise ratio in the velocity measurements may degrade the directional spreading estimates. Although the characteristics of the ADCP velocity noise are not well understood (see Hoitink et al. 2007), it is generally expected that noise reduces the coherence of the wave field across the array and thus may manifest itself in an overestimation of directional spreading, as is observed here.

The degraded signal-to-noise ratio of ADCP velocity measurements in benign wave conditions is also evident in the comparisons of significant wave height $H_{s}$ estimates based on velocity and pressure measurements (Fig. 3). The upper limit on the frequency range for these wave height estimates was restricted to $0.13 \mathrm{~Hz}$ to exclude waves with wavelengths shorter than twice the water depth (at the deeper ADCP site) that are strongly attenuated at the near-bottom pressure gauge. At both ADCP sites, there is a consistent positive bias in the velocity-based $H_{s}$ estimates relative to the pressure-based

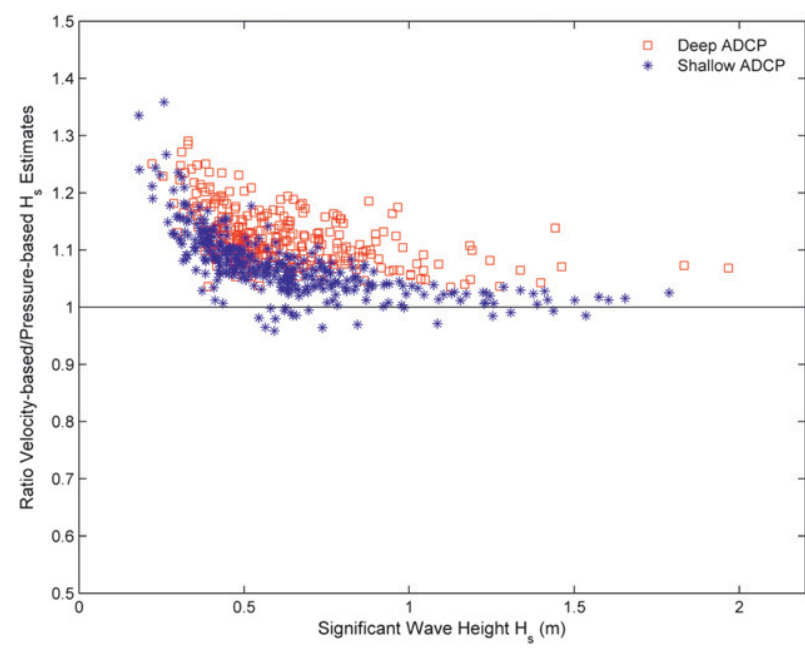

FIG. 3. Ratio between significant wave height estimates based on ADCP velocity and pressure measurements vs significant wave height (the pressure-based estimate). The frequency range of these estimates was restricted to $0.04-0.13 \mathrm{~Hz}$ to exclude strongly attenuated high-frequency wave components. The horizontal line indicates perfect agreement. The pressure-based estimates have errors (i.e., calibration inaccuracies) that are less than a few percent, and thus the observed bias (i.e., ratio values larger than 1) provides an indication of the relative noise level in the velocity measurements.

estimates. The apparent errors are notably larger for the deep ADCP, possibly because of the greater attenuation of the wave orbital velocity field and the longer acoustic ranges contributing to a lower signal-to-noise ratio. There is a clear dependence on wave energy levels; that is, at both ADCP sites, the bias in the $H_{s}$ estimates decreases with increasing wave height. For benign conditions $\left(H_{s} \approx 0.3-0.7 \mathrm{~m}\right)$, the deep ADCP velocitybased $H_{s}$ estimates are typically about $15 \%$ too high, and the shallow ADCP estimates are about $7 \%$ too high. For more energetic wave conditions $\left(H_{s} \approx 1-1.5 \mathrm{~m}\right)$, the typical bias in the deep ADCP estimates is reduced to about $8 \%$, whereas the shallow ADCP estimates are approximately unbiased. This dependence on sea state is consistent with the expectation of higher signal-to-noise ratios in larger waves with stronger orbital motions.

Detailed results for two representative case studies, a low-energy narrowband swell $\left(H_{s} \approx 0.6 \mathrm{~m}\right)$ observed on 27 October and a slightly more energetic $\left(H_{s} \approx 0.9 \mathrm{~m}\right)$ broader-band wave field observed on 10 November, are presented in Figs. 4 and 5, respectively. The 27 October case (Fig. 4) features a $0.06-\mathrm{Hz}$ swell with a slightly southerly (about $250^{\circ}$ ) direction and weaker higherfrequency waves from the west (about $280^{\circ}$ ). ADCPbased estimates of the mean wave direction $\bar{\theta}(f)$ agree well with the buoy-based estimates across the entire frequency spectrum. On the other hand, the directional 

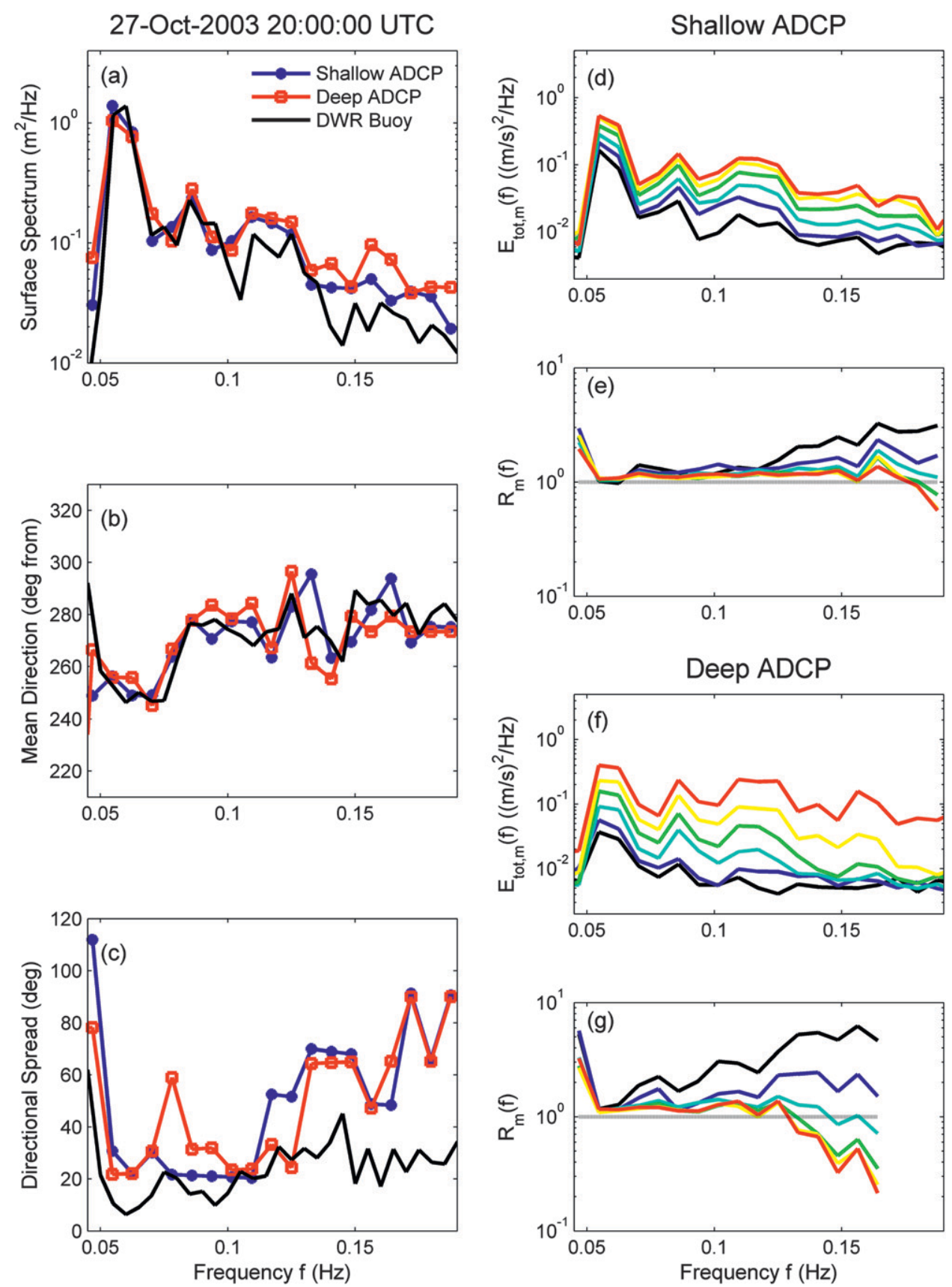

FIG. 4. Comparison of ADCP and Waverider buoy spectral estimates for a low-energy narrowband swell observed on 27 Oct 2003: (a) surface height spectral density, (b) mean wave direction, and (c) directional spread as functions of frequency. Solid black curves indicate estimates from the Waverider buoy. Symbols connected by colored lines denote estimates from the two ADCPs, transformed to the buoy depth using a frequency-dependent linear theory correction for shoaling and refraction effects. (d) The total velocity spectrum at each of the six sampled range bins of the shallow ADCP. The spectral levels decrease monotonically from the top bin (red curve) to the lowest bin (black curve). (e) The corresponding velocity-pressure transfer functions, normalized by the linear theory prediction (same color scheme). (f),(g) As in (d),(e), but for the deep ADCP. 

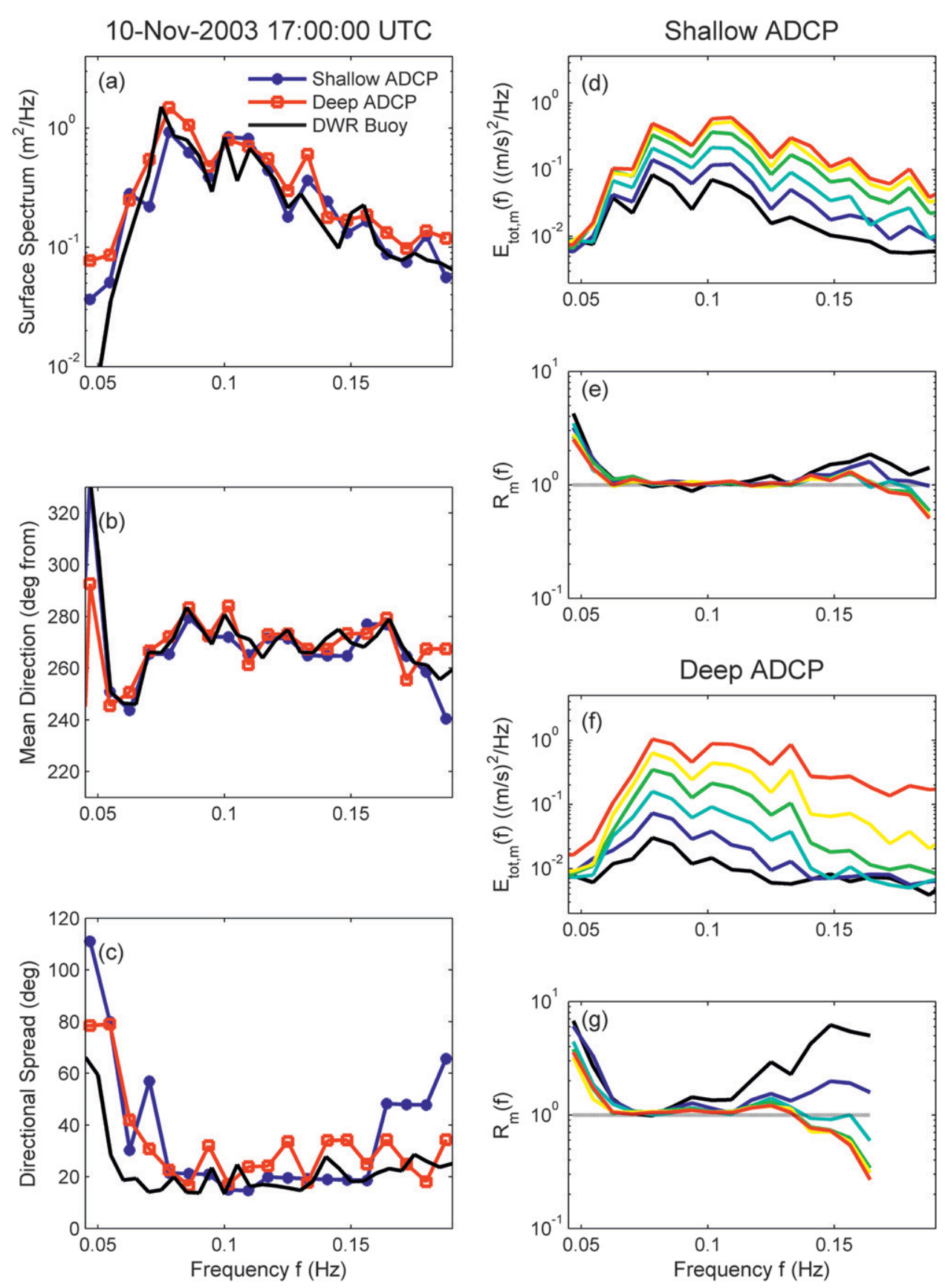

FIG. 5. As in Fig. 4, but for a moderately energetic, multimodal swell observed on 10 Nov 2003.

spreads $\sigma(f)$ are biased high. At the spectral peak, both ADCPs yield directional spreading values of about $20^{\circ}-30^{\circ}$ compared with the $5^{\circ}-10^{\circ}$ buoy estimates. At higher frequencies, the buoy-based $\sigma(f)$ estimates fluctuate between $20^{\circ}$ and $40^{\circ}$, whereas the ADCP-based estimates increase with increasing frequency to very large values (in excess of $60^{\circ}$ ) above $0.13 \mathrm{~Hz}$.

The bias in spectral energy levels and directional spreading estimates at high frequencies suggests high noise levels in the velocity measurements. To examine the quality of the velocity measurements at each of the six sampled depth cells, the sum of the four beam velocity spectra,

$$
E_{\mathrm{tot}, m}(f)=\sum_{n} C_{V_{n}^{m} V_{n}^{m}}(f),
$$

was computed, where the index $m$ (1-6) indicates the sampled cell and $n(1-4)$ is the beam. Because of the orthogonal beam configuration, $E_{\mathrm{tot}, m}$ is independent of 
the directional wave properties and decays monotonically with depth. The observations show the expected frequency-dependent decay that is stronger at the deeper site. At high frequencies, where the theoretical decay of the wave orbital motion is exponential, the velocity spectra of the lower depth cells collapse, indicating a noise floor of about $0.006\left(\mathrm{~m} \mathrm{~s}^{-1}\right)^{2} \mathrm{~Hz}^{-1}$. In particular, at the deeper ADCP, the spectra of the lowest four cells all collapse on this noise floor at frequencies above about $0.17 \mathrm{~Hz}$ (see section 5 for further discussion of the ADCP instrument noise floor).

Also shown in Fig. 4 is the corresponding ratio between measured velocity and pressure fluctuations, normalized by the theoretical linear transfer function

$$
R_{m}(f)=\frac{\omega \cosh \left(k d_{p}\right)}{g k \sqrt{2 \sin ^{2} \alpha \cosh ^{2}\left(k d_{m}\right)+4 \cos ^{2} \alpha \sinh ^{2}\left(k d_{m}\right)}} \sqrt{\frac{E_{\mathrm{tot}, m}(f)}{E_{p}(f)}}
$$

where $E_{p}$ is the pressure spectrum and $d_{p}$ is the height of the pressure gauge above the seafloor. In a linear wave field, the ratio $R_{m}$ is equal to 1 . Near the peak frequency, the observed ratios are close to 1 at all vertical levels. The errors are less than a few percent at the shallow ADCP, whereas the deep ADCP results show a small (about $10 \%$ ) positive bias. Above the peak frequency, the ratios diverge, with the largest values corresponding to the velocity measurements closest to the seafloor (the black curves), reflecting the decrease in signal-to-noise ratio with increasing frequency and depth. Closer to the surface, where the signal-to-noise ratio is high, the opposite trend is observed with $R_{m}$ falling off at high frequencies to values less than 1 (e.g., the red, yellow, and green curves for the deep ADCP in Fig. 4g). These observations are qualitatively consistent with the theoretically expected longer wavelength second-order bound wave contributions to the high-frequency wave motion that reduce the velocity/pressure transfer function (see Herbers et al. 1992 for more discussion).

The 10 November case (Fig. 5) features a more energetic wave field with a broad frequency spectrum. The dominant $(0.07-0.12 \mathrm{~Hz})$ swells arrived from westerly directions $\left(270^{\circ}-280^{\circ}\right)$ with a weak lower-frequency $(0.05-0.06 \mathrm{~Hz})$ swell from a more southerly direction $\left(240^{\circ}-250^{\circ}\right.$; indicative of a remote Southern Hemisphere source). Both the deep and shallow ADCP estimates of wave frequency spectra and mean wave directions capture this bimodality, as well as the finescale spectral structure in the buoy estimates. The directional spreading estimates from the shallow ADCP also agree well with the buoy estimates with values of about $20^{\circ}$ across the dominant swell frequency range. The deep ADCP estimates are slightly higher suggesting some contamination by instrument noise.

The velocity/pressure transfer functions for the 10 November case agree well with linear wave theory (Fig. 5). Estimates of the normalized velocity-pressure ratio $R_{m}$ [Eq. (16)] for the shallow ADCP are within $10 \%$ of the linear theory value 1 across a wide frequency range $(0.06-0.14 \mathrm{~Hz})$, with larger differences at lower and higher frequencies where the spectral levels are relatively low. The $R_{m}$ estimates for the deep ADCP are also in good agreement with linear theory near the spectral peak, where the spectral attenuation between the uppermost (red) and lowest (black) depth cells is almost two orders of magnitude. At frequencies above $0.08 \mathrm{~Hz}$, the ratios diverge with the largest errors in the lowest depth cell, similar to the 27 October case.

Overall, the present results show that a seafloormounted broadband ADCP, operated on the continental shelf in a standard wave-burst sampling mode, can provide reliable directional wave information. The main limitation appears to be the intrinsic noise level of the velocity measurements requiring (depending on the water depth) a moderately energetic wave field (i.e., significant wave height greater than $0.5 \mathrm{~m}$ in the swell conditions observed in this study) to resolve the attenuated velocity field over the entire water column. Whereas directional spreading estimates are sensitive to noise and tend to be biased high versus the buoy estimates, the mean wave direction estimates are accurate even in low $(<0.5 \mathrm{~m})$ wave conditions with marginal signal-to-noise ratios in the velocity measurements.

\section{Discussion}

The comparisons of ADCP directional spectrum estimates with buoy observations presented in the previous section generally show excellent agreement for the mean direction as a function of frequency across the entire swell band. On the other hand, the ADCP estimates of directional spread are biased high. To explain this bias, it is useful to note that directional spreading is inversely related to the spatial coherence; that is, the coherence length scale is infinite for a plane wave and vanishes in 


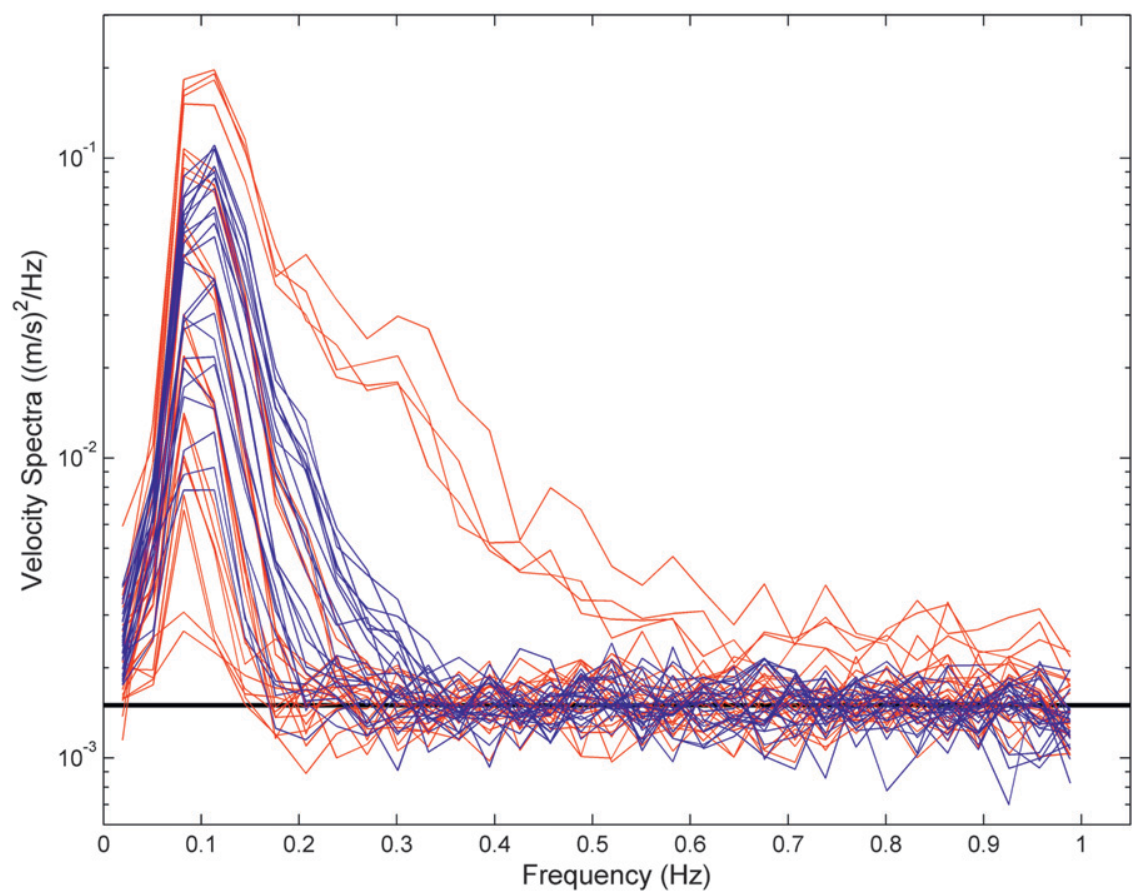

FIG. 6. Raw beam velocity spectra for the 10 Nov case observed at the deep (red curves) and shallow (blue curves) ADCP sites. The spectra were smoothed further by averaging four adjacent bands to identify the noise floor. All spectra with the exception of the near-surface cell of the deep ADCP (the top four red curves) collapse at high frequencies on a white noise spectrum with a level of about $0.0015\left(\mathrm{~m} \mathrm{~s}^{-1}\right)^{2} \mathrm{~Hz}^{-1}$ (black line).

the limit of an isotropic spectrum. The contribution of any noise to the measurements that is unrelated to the surface wave field will tend to reduce the spatial coherence scales and thus may cause a positive bias in directional spreading estimates.

In general there are three distinct sources of noise: 1) inaccuracies in the measurements usually referred to as instrument noise, 2) statistical uncertainty in the crossspectral estimates associated with finite record lengths, and 3) other geophysical contributions to the flow field. The latter source of noise may include, for example, highfrequency internal waves and turbulence associated with tidal and wind-driven currents. The spectral levels of these motions depend on the environment and are generally difficult to estimate. However, the spectra of the raw ADCP velocity time series (Fig. 6) roll off to a noise floor of about $0.0015\left(\mathrm{~m} \mathrm{~s}^{-1}\right)^{2} \mathrm{~Hz}^{-1}$, which is about one to two orders of magnitude higher than typical spectral levels of turbulent velocity fluctuations in coastal waters [e.g., vertical velocity spectra in similar depths reported by Shaw et al. (2001) and Gerbi et al. (2008)]. This noise level was observed consistently at both the deep and shallow sites throughout the experiment. Assuming a white noise spectrum over the entire frequency domain out to the $1-\mathrm{Hz}$ Nyquist frequency, this spectral level corresponds to a root-mean-square velocity fluctuation of about $4 \mathrm{~cm} \mathrm{~s}^{-1}$, which is somewhat smaller than the estimated $7 \mathrm{~cm} \mathrm{~s}^{-1}$ root-mean-square error in velocity estimates from a single acoustic ping (based on the RDI ADCP configuration program). Thus, the inherent random instrument noise appears to be a dominant source of errors in the ADCP velocity data.

To investigate the effect of ADCP instrument noise on wave directional spreading estimates, Monte Carlo simulations were performed for an idealized unimodal swell spectrum with a peak frequency of $0.1 \mathrm{~Hz}$ and a mean wave direction of $270^{\circ}$. Each simulation uses 1000 random plane wave components with the real and imaginary parts of the complex amplitudes taken from a standard normal distribution to create a Gaussian sea state. The frequency and propagation direction of each component were also taken from a normal distribution with mean (standard) deviation of $0.1(0.015) \mathrm{Hz}$ and $270^{\circ}\left(10^{\circ}\right)$ to simulate a wave field with realistic frequency and direction bandwidths. The directional spread, defined in the method presented here as the half-width of the directional distribution at half-maximum power, is $11.8^{\circ}$, which is close to the $10^{\circ}$ standard deviation of the Gaussian-shaped directional distribution. The ADCP velocity time series were created from the simulated 
surface elevation field using the linear wave theory transfer functions (5). Added to these wave velocity records was uncorrelated Gaussian noise with a standard deviation of $4 \mathrm{~cm} \mathrm{~s}^{-1}$ to represent the observed instrument noise. The record length and sampling interval of the simulated data records are the same as those of the actual data records to faithfully reproduce the statistical uncertainty of finite-length records and spectral noise characteristics.

A total of 500 simulations were performed for both the deep and shallow ADCP configurations with the significant wave height varying from 0.2 to $2 \mathrm{~m}$ to examine the effect of instrument noise on varying sea states. Estimates of the mean wave direction and directional spread as functions of frequency were extracted from the simulated ADCP data using exactly the same processing code as was applied to the field data. Estimates of these parameters at the spectral peak frequency are shown as a function of the significant wave height in Fig. 7. Estimates of the mean wave direction (Fig. 7a) are close to the true direction of $270^{\circ}$, even for very small wave heights. This insensitivity is expected, because the simulated white instrument noise is directionally isotropic and thus does not introduce a directional bias. On the other hand, the directional spread estimates (Fig. 7b) are clearly affected by the instrument noise with a positive bias that increases with decreasing wave height (i.e., decreasing signal-to-noise ratio). For benign conditions with significant wave heights less than $0.4 \mathrm{~m}$, this bias is appreciable (estimated spread values ranging from $15^{\circ}$ to very large values), roughly consistent with the errors inferred from the field data (Fig. 2a,d). Although the simulations presented here use an idealized wave field and do not account for other potential sources of noise that may not be spatially incoherent or isotropic, it is clear that the inherent relatively large instrument noise levels of the ADCP can degrade the quality of wave directional spreading estimates in benign wave conditions.

\section{Summary and conclusions}

Acoustic Doppler current profilers (ADCPs) are widely used to collect routine measurements of current profiles and surface wave spectra in coastal waters. The accuracy of directional wave measurements obtained with a seafloor-mounted ADCP was examined in this study using two standard broadband $600-\mathrm{kHz}$ instruments deployed in 20- and 45-m depths on the Southern California continental shelf. The ADCP with four upward-looking beams in a Janus configuration sampled the wave-orbital velocity field at six vertical levels spanning the water column, forming a 24-element
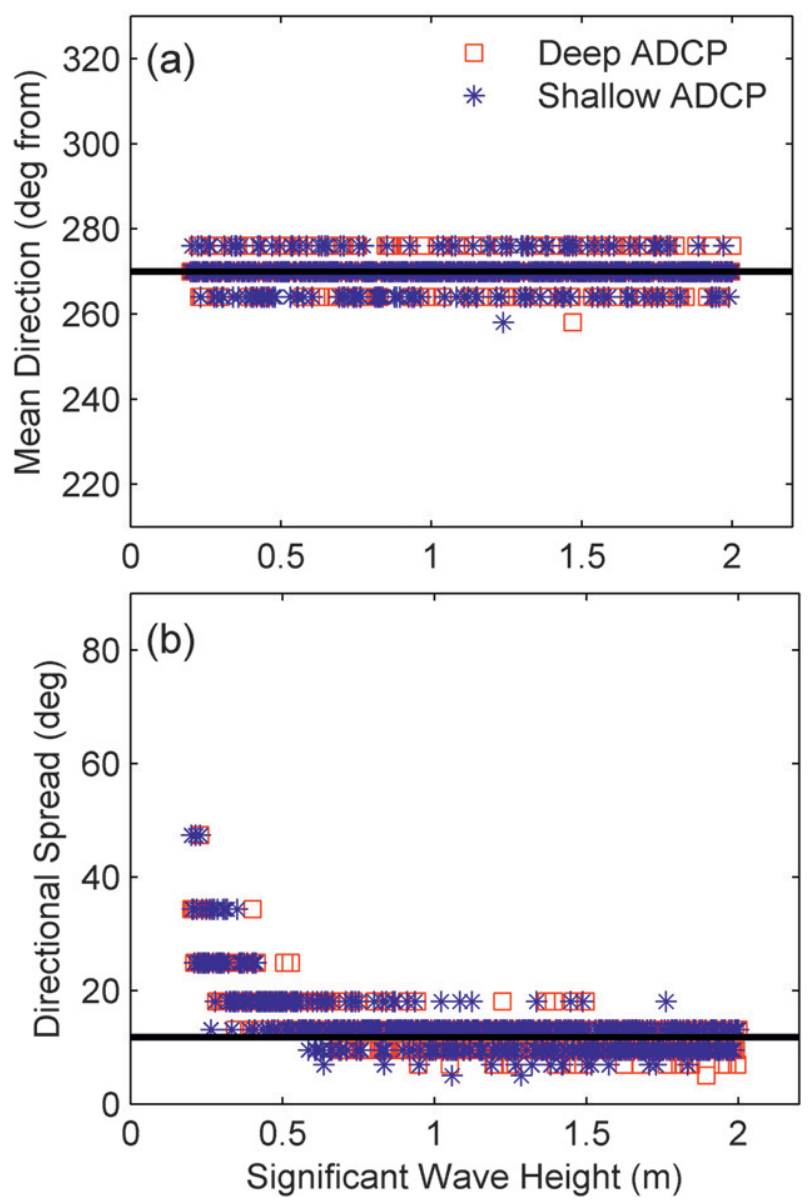

FIG. 7. Simulation of the effect of random instrument noise on directional spread estimates. Each symbol represents a Monte Carlo simulation of a Gaussian swell field (spectral peak at $0.1 \mathrm{~Hz}$ and $270^{\circ}$ ) with realistic spectral widths in frequency and direction. Uncorrelated Gaussian noise with a standard deviation of $4 \mathrm{~cm} \mathrm{~s}^{-1}$ (approximately the noise level of the ADCP) was added to each of the velocity time series. (a) Estimated mean wave direction and (b) directional spread (both at the peak frequency) for a range of significant wave heights. Results for the deep and shallow ADCP sites are indicated with red squares and blue asterisks, respectively. Solid lines indicate the true mean wave direction and directional spread.

coherent array. A simple parametric technique is presented that fits a cosine-power unimodal or bimodal directional distribution of wave energy to the array crossspectra. Fitting such a model with only a few degrees of freedom has the advantage that robust estimates of the mean direction and spreading of one or two dominant modes of wave energy may be extracted from the inherently noisy velocity measurements. More complex solutions to this poorly constrained inverse problem are thus rejected to avoid the overinterpretation of possibly spurious multimodal features. In fact, the bimodal model did not significantly improve the fit to the 
array cross-spectra, possibly because the directional aperture of swell arrivals is too narrow at this near-shore field site, sheltered by islands in the Southern California Bight, to resolve crossing wave trains at the same frequency in the ADCP data. Therefore, only the unimodal directional distribution estimates were used in this study.

ADCP estimates of the mean wave direction and directional spread as functions of frequency were compared with estimates from a nearby deployed Datawell Directional Waverider buoy. Wave conditions during the experiment ranged from the prevalent low (about 0.5 -m significant wave height) background swell to moderately energetic (1-2 m) events. ADCP-based estimates of mean wave directions are in excellent agreement with the buoy estimates. Even in very low $(<0.5 \mathrm{~m})$ wave conditions, both the deep and shallow ADCPs accurately resolve the usually subtle variations in mean wave directions across the spectrum. On the other hand, ADCP-based estimates of directional spread are biased high. The agreement with buoy results is notably better for the shallow ADCP than the deep ADCP, and errors appear to depend on the sea state with a smaller bias in more energetic wave conditions. These results are consistent with degraded signal-to-noise ratios expected in low-energy wave conditions and deeper water (i.e., longer acoustic ranges and the hydrodynamic attenuation of the wave orbital motion with depth).

The importance of noise in the velocity measurements is confirmed by an analysis of the spectral transfer functions between ADCP velocity and pressure measurements. Comparisons of the observed transfer functions with linear wave theory show a clear dependence on sea state with a positive bias that decreases with increasing wave height. This bias, indicative of noise contributions to the velocity spectra, is consistently about a factor of 2 smaller for the shallow ADCP (errors usually less than $10 \%$ in the energetic part of the spectrum) than for the deep ADCP.

Noise in the velocity measurements does not appear to affect estimates of mean wave direction, but it significantly degrades estimates of directional spreading. This sensitivity is consistent with the effect of random, uncorrelated noise that reduces the horizontal coherence of the velocity measurements in much the same way that increased directional spreading reduces the coherence. Monte Carlo simulations of the effect of uncorrelated Gaussian noise on ADCP measurements of idealized swell conditions confirm that the random uncertainty in the Doppler shift estimates of acoustic pings contributes a dominant source of error in the wave directional spreading estimates.

In summary, the present results show that a standard $600-\mathrm{kHz}$ broadband ADCP can provide robust ocean surface wave information on the continental shelf. Whereas the wave energy spectrum and dominant wave directions are accurately measured by the instrument, directional spreading is generally overestimated as a result of the coherence reducing effect of random instrument noise, especially when the signal-to-noise ratio is degraded in benign wave conditions and/or deeper deployment depths. A comparison of ADCP velocity and pressure spectra with the linear theory transfer function [Eq. (16); Figs. 4e,g, 5e,g] and estimates of the relative noise level in the raw beam spectra (Fig. 6) can provide useful diagnostic checks on the quality of the data and help to identify the frequency range over which the wave orbital motion is resolved. Overall, the ADCP, with its dual capability of measuring ocean currents and waves, is an attractive instrument for collecting routine directional wave information in coastal applications.

Acknowledgments. This research was supported by the National Science Foundation (Physical Oceanography Program) and the Office of Naval Research (Coastal Geosciences Program). The instruments were deployed and maintained by staff from the Scripps Institution of Oceanography and the Naval Postgraduate School. We thank Julie Thomas and Bill O'Reilly for collecting and archiving the Waverider buoy observations and Paul Jessen and Jeremy Boyd for their help with the ADCP data analysis. We also appreciate helpful suggestions by the anonymous reviewers.

\section{REFERENCES}

Ardhuin, F., T. H. C. Herbers, P. F. Jessen, and W. C. O'Reilly, 2003: Swell transformation across the continental shelf. Part II: Validation of a spectral energy balance equation. J. Phys. Oceanogr., 33, 1940-1953.

Brumley, B. H., R. G. Cabrera, K. L. Deines, and E. A. Terray, 1991: Performance of a broad-band acoustic Doppler current profiler. IEEE J. Oceanic Eng., 16, 402-407.

Gerbi, G. P., J. H. Trowbridge, J. B. Edson, A. J. Plueddemann, E. A. Terray, and J. J. Fredericks, 2008: Measurements of momentum and heat transfer across the air-sea interface. J. Phys. Oceanogr., 38, 1054-1072.

Hasselmann, D. E., M. Dunckel, and J. A. Ewing, 1980: Directional wave spectra observed during JONSWAP 1973. J. Phys. Oceanogr., 10, 1264-1280.

Herbers, T. H. C., and R. T. Guza, 1990: Estimation of directional wave spectra from multicomponent observations. J. Phys. Oceanogr., 20, 1703-1724.

—, R. L. Lowe, and R. T. Guza, 1991: Field verification of acoustic Doppler surface gravity wave measurements. J. Geophys. Res., 96 (C9), 17 023-17035.

,-- , and -1992 : Field observations of orbital velocities and pressure in weakly nonlinear surface gravity waves. J. Fluid Mech., 245, 413-435. 
— , S. Elgar, and R. T. Guza, 1994: Infragravity-frequency $(0.005-0.05 \mathrm{~Hz})$ motions on the shelf. Part I: Forced waves. J. Phys. Oceanogr., 24, 917-927.

$[,-$, and 1999 : Directional spreading of waves in the nearshore. J. Geophys. Res., 104 (C4), 7683-7693.

Hoitink, A. J. F., H. C. Peters, and M. Schroevers, 2007: Field verification of ADCP surface gravity wave elevation spectra. J. Atmos. Oceanic Technol., 24, 912-922.

Krogstad, H. E., R. L. Gordon, and M. C. Miller, 1988: Highresolution directional wave spectra from horizontally mounted acoustic Doppler current meters. J. Atmos. Oceanic Technol., 5, 340-352.

Kuik, A. J., G. Ph. van Vledder, and L. H. Holthuijsen, 1988: A method for the routine analysis of pitch-and-roll buoy wave data. J. Phys. Oceanogr., 18, 1020-1034.

Lhermitte, R., and R. Serafin, 1984: Pulse-to-pulse coherent Doppler sonar signal processing techniques. J. Atmos. Oceanic Technol., 1, 293-308.

Lippmann, T. C., T. H. C. Herbers, and E. B. Thornton, 1999: Gravity and shear wave contributions to nearshore infragravity motions. J. Phys. Oceanogr., 29, 231-239.

Long, R. B., and K. Hasselmann, 1979: A variational technique for extracting directional spectra from multi-component wave data. J. Phys. Oceanogr., 9, 373-381.

Longuet-Higgins, M. S., D. E. Cartwright, and N. D. Smith, 1963: Observations of the directional spectrum of sea waves using the motions of a floating buoy. Ocean Wave Spectra, PrenticeHall, 111-136.

Magne, R., K. A. Belibassakis, T. H. C. Herbers, F. Ardhuin, W. C. O'Reilly, and V. Rey, 2007: Evolution of surface gravity waves over a submarine canyon. J. Geophys. Res., 112, C01002, doi:10.1029/2005JC003035.

Mitsuyasu, H., F. Tasai, T. Suhara, S. Mizuno, M. Ohkusu, T. Honda, and K. Rikiishi, 1975: Observations of the directional spectrum of ocean waves using a cloverleaf buoy. J. Phys. Oceanogr., 5, 750-760.
Munk, W. H., G. R. Miller, F. E. Snodgrass, and N. F. Barber, 1963: Directional recording of swell from distant storms. Philos. Trans. Roy. Soc. London, A255, 505-584.

O'Reilly, W. C., T. H. C. Herbers, R. J. Seymour, and R. T. Guza, 1996: A comparison of directional buoy and fixed platform measurements of Pacific swell. J. Atmos. Oceanic Technol., 13, 231-238.

Pawka, S. S., 1983: Island shadows in wave directional spectra. J. Geophys. Res., 88 (C4), 2579-2591.

Pinkel, R., 1979: Observations of strongly nonlinear internal motion in the open sea using a range-gated Doppler sonar. J. Phys. Oceanogr., 9, 675-686.

- 1983: Doppler sonar observations of internal waves: Wavefield structure. J. Phys. Oceanogr., 13, 804-815.

- , and J. A. Smith, 1987: Open ocean surface wave measurement using Doppler sonar. J. Geophys. Res., 92 (C12), 12 96712973.

Shaw, W. J., J. H. Trowbridge, and A. J. Williams III, 2001: Budgets of turbulent kinetic energy and scalar variance in the continental shelf bottom boundary layer. J. Geophys. Res., 106 (C5), 9551-9564.

Smith, J. A., 1993: Performance of a horizontally scanning Doppler sonar near shore. J. Atmos. Oceanic Technol., 10, 752-763. , and J. L. Largier, 1995: Observations of nearshore circulation: Rip currents. J. Geophys. Res., 100 (C6), 10 967-10 975.

Terray, E. A., B. H. Brumley, and B. Strong, 1999: Measuring waves and currents with an upward-looking ADCP. Proc. Sixth Working Conference on Current Measurement, San Diego, IEEE, 66-71.

Thomson, J., S. Elgar, T. H. C. Herbers, B. Raubenheimer, and R. T. Guza, 2007: Refraction and reflection of infragravity waves near submarine canyons. J. Geophys. Res., 112, C10009, doi:10.1029/2007JC004227.

Work, P. A., 2008: Nearshore directional wave measurements by surface-following buoy and acoustic Doppler current profiler. Ocean Eng., 35, 727-737. 